\title{
Postsozialistische Stadtentwicklungen zwischen nachholender Modernisierung und eigenem Weg
}

\section{Joachim Burdack, Robert Rudolph, Leipzig}

\section{Einführung}

"Wer derzeit in den Städten des östlichen und mittleren Europa unterwegs ist, traut seinen Augen nicht. Die Intervalle, in denen sich Städte ändern, werden immer kürzer... Ideen und Reiche vergehen, aber nicht der Ort.»,

schrieb der Historiker KaRL SCHLÖgEL 1997. Insbesondere die Großstädte Ostmittel- und Osteuropas werden seit dem Zerfall der zentral gesteuerten Regulationsmechanismen der Wirtschaft und Gesellschaft nach dem Ende des Sozialismus von tiefgreifenden Veränderungen erfasst. Die neue Bedeutung der Städte als Orte, die globalen Einflüssen intensiv ausgesetzt und daher für strukturelle Wandlungsprozesse besonders prädestiniert sind, wird durch die schnelle ökonomische Entwicklung einiger Metropolen deutlich. Städte, die bis dahin lediglich Zentrum eines relativ isolierten Umlandes waren, entwickeln neue Funktionen als nationale oder regionale gateways; zuvor peripher gelegene Grenzregionen befinden sich plötzlich in begünstigter Nähe zu den ökonomischen Kernräumen Westeuropas, ehemalige Industrieregionen sind von massiven Deindustrialisierungsprozessen betroffen.

Der Versuch, die postsozialistischen Entwicklungen theoretisch zu fassen, führte zunächst $\mathrm{zu}$ einem Rückgriff auf modernisierungstheoretische Ansätze. Insbesondere die ökonomischen Theorien fassten die Transformation weitgehend als eindimensionalen Prozess, in erster Linie als Koordinierungsproblem und bestenfalls als Investitionsprozess auf (vgl. u.a. KLEIN 1995, GöTz 1998a). Der Begriff «Transformation», der als paradigmatischer Leitbegriff geographischer Forschung im östlichen Europa dient, wird in der Literatur nicht eindeutig verwendet. Im Sinne von FasSMANN (1997) bezeichnet Transformation den umfassenden und aus historischer Perspektive außerordentlich schnellen Wandel der politischen, ökonomischen und sozialen Strukturen der ostmitteleuropäischen und osteuropäischen, ehemals sozialistischen Gesellschaften seit dem Ende der 1980er Jahre. In den Modellvorstellungen einer «nachholenden Modernisierung» wird implizit ein bekanntes Endziel angenommen, lediglich die Zeitspanne der Transformation sei unbekannt. Übertragen auf die Stadtentwicklung leitete sich hieraus die Erwartung des Nachholens von Prozessen $a b$, die westliche Städte in den letzten Jahrzehnten geprägt haben. Inzwischen erscheint es zweifelhaft, ob der Umbruch der osteuropäischen Planwirtschaften als Transformation mit bekanntem Ausgang interpretiert werden kann. SzELENYI (1996) formulierte z.B. die These, dass postsozialistische Städte möglicherweise nicht westlichen Entwicklungsmustern folgen, sondern Stadtentwicklungsprozessen ähneln könnten, die für Entwicklungsländer typisch sind.

Der folgende Beitrag untersucht den Wandel von Städten im östlichen Europa anhand von vier Fallbeispielen und geht dabei der Frage nach, ob unterschiedliche Entwicklungspfade postsozialistischer Stadtentwicklung erkennbar sind. Die Auswahl der Fallstudien Moskau, Budapest, Jekaterinburg und Łódź berücksichtigt zwei Differenzierungsachsen postsozialistischer Stadtentwicklung: zum einen sollen die unterschiedlichen Entwicklungen funktionaler Stadttypen, d.h. von Hauptstädten bzw. Primatstädten und relativ monostrukturierten Industriestädten untersucht werden, zum anderen ist die regionale Differenzierung zwischen Städten in Ländern mit fortgeschrittener und solchen mit «instabiler» Transformation Gegenstand der Betrachtung.

Komplexe Systeme wie Städte können nicht in ihrer Totalität miteinander verglichen werden, sondern nur hinsichtlich bestimmter Merkmale. Es ist deshalb notwendig, die Merkmale zu spezifizieren, die der jeweiligen Analyse zugrunde liegen. Die vergleichende Analyse kann entweder als bottom up-Ansatz aus den Fallstudien selbst entwickelt werden («grounded theory»), oder sie wird als top down-Ansatz aus theoretischen Stadtentwicklungsdiskursen abgeleitet. Beide Vorgehensweisen haben spezifische Stärken und Schwächen.

Der bottom up-Ansatz trägt der Individualität der untersuchten Städte Rechnung, führt aber oft zu Aussagen, die nur eine begrenzte Passfähigkeit bei der Einordnung in größere Prozesszusammenhänge besitzen. Diese Vergleichbarkeit gewährleistet ein top downAnsatz. Er unterliegt jedoch seinerseits der Gefahr, dass die «von außen» zugrunde gelegten Kriterien den Untersuchungsgegenstand in ein starres begriffliches Korsett pressen, was zu erheblichen Verzerrungen führen kann. Die vorliegende Studie folgt, bedingt durch die übergeordnete Fragestellung, eher einer top down-Strategie. Den damit verbundenen Schwächen wird jedoch durch die Berücksichtigung der jeweiligen handlungsstrukturierenden Rahmenbedingungen entgegengewirkt.

Die Städte der sozialistischen Gesellschaften wiesen - unterschiedlichen regionalen Ausprägungen und verschiedenen Leitbildern folgend - sehr spezifische Merkmale auf, die heute zu den entscheidenden 
Bestimmungsfaktoren des postsozialistischen Wandels zählen. Dieser Wandel wird insbesondere anhand strukturbildender Prozesse wie Tertiärisierung, Deindustrialisierung, Suburbanisierung und Gentrifizierung/ Reurbanisierung untersucht. Es wird dabei der Frage nachgegangen, in welchem Umfang und mit welchen resultierenden Raummustern sich die Prozesse, die die Entwicklung westlicher Städte in den letzten Jahrzehnten maßgeblich bestimmt haben, auch in den postsozialistischen Untersuchungsstädten feststellen lassen. In diesem Kontext können Entwicklungen, die einem westlichen Muster folgen, als Beleg für «nachholende» Prozesse gewertet werden, während auf der anderen Seite solche, die deutlich abweichende Prozessverläufe zeigen, als Ausdruck eigener Entwicklungspfade verstanden werden.

\section{Die «sozialistische Stadt» aus historischer Perspektive}

Der Begriff «sozialistische Stadt» ist mehrdeutig. $\mathrm{Zu}$ unterscheiden ist das weltanschauliche Modell der «sozialistischen Stadt» und die Realität der Stadt im Sozialismus in ihren verschiedenen Entwicklungsphasen (vgl. Karger \& Werner 1982). Die Stadtentwicklung im östlichen Europa ließ auch unter sozialistischen Bedingungen ein breites Spektrum unterschiedlichster Erscheinungen erkennen. So sind etwa seit den 1970er Jahren vor allem in Ungarn und zum Teil in Polen deutliche Divergenzen zum «sowjetischen Entwicklungsmodell» erkennbar. Trotz dieser Einschränkungen lassen sich länderübergreifende Merkmale und Prozesse feststellen, die die Entwicklung der Städte in den sozialistischen Staaten des östlichen Europa bestimmt haben (French \& Hamiltion 1979, Smith 1989, Musil 1993, Andrusz, Harloe \& Szelenyi 1996).

Als theoretische Leitvorstellung der sozialistischen Stadtentwicklung kann die Schaffung gleichwertiger Lebensbedingungen und eine Einebnung der Unterschiede im städtischen Lebensniveau angesehen werden (HäussermanN 1997). Die realen städtebaulichen, funktional- und sozialräumlichen Strukturen der Städte waren jedoch weniger Ausdruck zielgerichteten planerischen Handelns, sondern vor allem Ergebnis nicht-intendierter Handlungsfolgen der sozialistischen Planung und letztendlich Ausdruck immanenter Widersprüche des «real existierenden Sozialismus». Zwar beruhten in den sozialistischen Gesellschaften wesentliche Stadtentwicklungsprozesse auf einer zentralen Planung und Standortentscheidungen staatlicher Stellen, jedoch war es auch unter sozialistischen Bedingungen in einem so komplexen sozialen und ökonomischen System, wie es Städte darstellen, nicht möglich, alle Prozesse normativ zu planen und zu steuern (vgl. ENYEDI 1994). Die entstehenden räumlichen Strukturen der städtischen Flächennutzung beruhten in den mittel- und osteuropäischen Planwirtschaften nicht auf Marktmechanismen, dennoch waren die Standortscheidungen im wesentlichen rationalen ökonomischen Zwängen unterworfen.

Nach SzelenYI (1996) ist das charakteristische Merkmal der «sozialistischen Stadt» nicht ein bestimmtes Erscheinungsbild oder eine Zielvorstellung sozialistischer Planer, sondern die Tatsache, dass es sich um Städte industrialisierter Gesellschaften handelt, die den Privatbesitz von Produktionsmitteln abgeschafft haben. Als wichtige Faktoren einer spezifisch sozialistischen Stadtentwicklung seien folgende genannt:

- Städtischer Grund und Boden war entweder verstaatlicht oder zumindest dem privaten Verfügungsrecht weitgehend entzogen. Es existierte kein Preisbildungsmechanismus für Grundstücke. Der Lagerente kommt, durch das Fehlen entsprechender Bodenpreisgradienten, keine bestimmende Rolle bei der Nutzungsdifferenzierung zu.

- Der aus ökonomischen und ideologischen Gründen forcierte Ausbau der Schwerindustrie und die Nutzung der vorhandenen Agglomerationsvorteile führten zwangsläufig zum Wachstum der großen Städte und Industriezentren. Großflächige neue Industrieareale mit überdimensionierten Betriebsflächen und Flächenreserven entstanden. Andererseits lässt sich auch eine Weiternutzung der alten Industriegebiete der vorsozialistischen Zeit feststellen, da fehlender Umnutzungsdruck und Kapitalmangel eine Standortpersistenz begünstigten.

- Die Kehrseite des massiven Ausbaus der Schwerindustrie waren die dauerhaft geringen Investitionen in nichtproduktiven Bereichen. Die generelle Unterentwicklung und Unterrepräsentanz des als unproduktiv geltenden tertiären Sektors in den sozialistischen Gesellschaften, insbesondere dessen konsumorientierte Bereiche, wurde so zu einem weiteren Merkmal sozialistischer Städte (vgl. Bater 1980, Karger \& Werner 1982, ENyedi 1994).

- Wohnen sollte im Sozialismus keine Ware mehr sein. Ein Instrument, das die Stadtplanung dazu einsetzte, war die bewusste Aufhebung von Segregation in der Stadtbevölkerung durch Standardisierung des Wohnungsbaus und staatliche Regulation des Wohnungsmarkts (Finanzierung, Erstellung, Zuteilung, Verwaltung, Instandhaltung) (MusIL 1993, RUDOLPH \& LenTZ 1999). Neue vielgeschossige Großwohngebiete entstanden in den Außenbezirken, das flächenhafte Stadtwachstum an der Peripherie wurde zum dominanten räumlichen Entwicklungsmuster. Durch das weitgehende Fehlen privater Bautätigkeit kam es dagegen nicht zu einer Ausbildung von Einfamilienhausgürteln in der Form von urban sprawl. Unterschiedliche nationale Wohnungspolitiken führten seit den 1970er Jahren jedoch zu deutlich unterschiedlichen Wohneigentumsquoten (KovÁcs 1997). 
- Übereinstimmend stellen Untersuchungen fest (z.B. Dangschat 1987, Hamilton \& French 1979), dass auch die Städte im Sozialismus von sozialen Segregationserscheinungen geprägt waren. SzELENYI (1996) betont, dass die Segregationsmuster sozialistischer Städte nicht Überbleibsel der «kapitalistischen Vergangenheit» waren, sondern als ein Ergebnis sozialer Widersprüche innerhalb der sozialistischen Gesellschaften interpretiert werden müssen. Entgegen aller theoretischen Ziele existierte durchaus eine starke soziale Differenzierung, sie äußerte sich sozialräumlich u.a. in der Praxis einer privilegierenden Zuweisung von Wohnraum. Segregation orientierte sich weniger an Einkommensunterschieden, sondern an einem «vielfältig ausdifferenzierten System gesellschaftlicher Privilegierung und Diskriminierung» (HäUSSERMANN 1997: 24).

Viele Modelle der «sozialistischen Stadt» als geographischem Stadtstrukturtyp beziehen sich auf physiognomische Merkmale der Architektur und des Städtebaus, die typische «sozialistische» Gestaltungselemente darstellen. Im Rahmen verschiedener Leitbilder entwickelte der sozialistische Städtebau charakteristische Strukturelemente, wie die «Magistrale» und den «Zentralen Platz», mit deren Hilfe sich ein Stadtmodell im kulturhistorischen Sinne entwikkeln ließ (Karger \& Werner 1982: 519ff.). Konsequent zur Anwendung kamen diese Strukturelemente jedoch in erster Linie in den Städten, die nach den Prinzipien des sozialistischen Städtebaus tatsächlich neu gebaut wurden bzw. in jenen Stadtteilen, die durch die flächenhafte Beseitigung älterer Bausubstanz und Neubebauung stark überprägt worden sind.

\section{Städtische Strukturen in der Transformation}

\subsection{Der Wandel der Städte im östlichen Europa}

Wir finden innerhalb des östlichen Europa eine Pluralität der Transformation: zwischen den Staaten, Regionen und Städten vervielfältigen sich die politischen, ökonomischen und sozialen Bedingungen als auch die Handlungsstrategien der Akteure auf nationaler, regionaler und lokaler Ebene.

In ökonomischer Hinsicht ist der mit der Transformation verbundene Strukturwandel der Städte durch einen doppelten Anpassungsprozess gekennzeichnet:

- die zentral gesteuerte planwirtschaftliche Ökonomie wird allmählich durch dezentralisierte marktwirtschaftliche Strukturen ersetzt,

- die ehemals sozialistischen Staaten sind nach ihrer Öffnung den globalen Einflüssen ausgesetzt, die weltweit wirtschaftsstrukturelle Veränderungen hervorrufen und in ihrer Folge $\mathrm{zu}$ innerstädtischen Strukturveränderungen führen.

Der Transformationsprozess vollzieht sich somit im
Spannungsfeld von interner Restrukturierung und zunehmender Internationalisierung. Während die Städte unter sozialistischen Bedingungen sehr stark in den jeweiligen, vergleichsweise abgeschlossenen nationalen Wirtschaftsraum integriert und funktionale Zentren eines entsprechenden, staatlich festgelegten Einzugsbereiches waren, sind sie nun in eine Vielzahl globaler, nationaler und regionaler Zusammenhänge eingebunden. Einerseits eröffnen sich vor dem Hintergrund gesamteuropäischer Integrationsprozesse für die Städte der ehemals sozialistischen Länder Möglichkeiten, neue internationale Verbindungen aufzubauen, neue ökonomische Funktionen und Bedeutungen im Städtesystem zu entwickeln. Mit der Transformation und Demokratisierung ist auch eine Verlagerung von Entscheidungsprozessen auf die regionale und lokale Ebene verbunden. Die großen Städte können nunmehr als Handlungsakteure und Entscheidungsträger selbstständig grenzüberschreitende Beziehungen aufbauen. Auf der anderen Seite ergeben sich durch die Errichtung neuer Grenzen und Barrieren auch neue periphere Situationen, die sich auf die ökonomische und soziale Entwicklung der Stadtregionen auswirken können. Vor allem bedeutet die Öffnung der Grenzen und die Integration ostmittel- und osteuropäischer Ökonomien in den Weltmarkt die Entstehung einer europäischen bzw. globalen Standortkonkurrenz, denen die Städte dieses Raumes ausgesetzt sind.

Polarisierungstendenzen wurden von stadtgeographischer Seite (u.a. Grimm 1994, Fassmann \& LichtenBERGER 1995, FASSMANN 1997) als länderübergreifende Transformationsphänomene beschrieben. Hierbei bestätigte sich, dass insbesondere die Hauptstädte der Transformationsländer starken Veränderungen unterworfen sind. Die zunehmenden räumlichen Disparitäten innerhalb der osteuropäischen Staaten erwiesen sich als Ungleichzeitigkeiten der Transformation in verschiedenen Regionstypen. Der Übergang zu neuen politischen und wirtschaftlichen Strukturen verlief am frühesten und intensivsten in den großen Metropolen in Ostmittel- und Osteuropa, wie Budapest, Prag, Warschau, Riga, Kiew und Moskau. Nach Grimm (1994) werden die Großstädte mit Hauptstadtfunktion «mit hoher Wahrscheinlichkeit die führenden Steuerungszentralen und Träger der künftigen mittel- und osteuropäischen Raumentwicklung sein.» (ebd.: 25).

\subsection{Postsozialistische Stadtentwicklungen Moskau - Hauptstadt Russlands und postsowjetische Metropole}

Moskau bildete hinsichtlich seiner regionalen Wirtschaftsstruktur immer eine Besonderheit unter den sowjetischen Städten. Die Stadt war einer vergleichsweise intensiven Tertiärisierung ausgesetzt, wobei insbesondere der wissenschaftliche, wirtschaftsleitende und administrative Sektor stark dominierte. Bereits 
wenige Jahre nach dem Beginn der Reformen hatte Moskau eine außergewöhnliche Position im wirtschaftlichen Entwicklungsprozess erreicht. Es zeigte sich sehr schnell, dass Moskau trotz der «offiziellen» Funktionsverluste seine Position im nationalen Städtesystem festigen und ausbauen konnte (vgl. STADELBAUER 1996, Kolossow et al. 1998). In Moskau, einer Stadt mit heute 8,5 Mill. Einwohnern, trafen die Transformationsprozesse auf ein städtisches System, das bereits vor Beginn der politischen Öffnung und ökonomischen Liberalisierung durch einen strukturellen Vorsprung gegenüber anderen russischen Großstädten gekennzeichnet war. Moskau hatte daher weitaus bessere Ausgangsbedingungen, sich schneller und erfolgreicher den Bedingungen der global economy anzupassen als andere Landesteile. Moskau erfuhr während der 1990er Jahre ein räumlich selektives ökonomisches Wachstum. Absolut dominant hinsichtlich der Kapitalinvestitionen ist bis heute das Stadtzentrum.

Ein offensichtliches Merkmal postsozialistischer Stadtentwicklung in Moskau ist die Bildung einer differenzierten «Geschäftscity» mit funktionalen, städtebaulichen und architektonischen Formen, die sich an internationalen Citystrukturen orientieren (Entwicklung gehobener Geschäftsstraßen in der Innenstadt, Entwicklung von Bürogebäuden mit «internationalem Standard», von Bürovillen, Hotels, Passagen). Der Transformationsprozess äußert sich hier in einer außergewöhnlich schnellen Citybildung (vgl. LENTZ 1997, 2000, Rudolph 1997). Daneben sind in Moskau neue sozialräumliche Differenzierungen zu beobachten, wobei insbesondere das Stadtzentrum und einige stalinzeitliche Straßenachsen als bevorzugte Wohnstandorte für die neue russische Oberschicht in Erscheinung treten. Den neu entstehenden Raumstrukturen liegen veränderte Steuerungsmechanismen der Stadtentwicklung zugrunde. Insbesondere die Privatisierung des Gebäude- und Wohnungsbestandes spielt hierbei eine bedeutende Rolle. Mit der Entstehung eines Immobilienmarktes, der sich bisher vorwiegend auf der Grundlage des Handels mit Pachtrechten an Grundstücken und Nutzungsrechten an Wohnungen und Gebäuden entwickelt, wird der Standort innerhalb der Stadt zunehmend zu einer monetär bewerteten Ware (vgl. Abb. 1).

Charakteristische Merkmale der sowjetischen Stadt waren Unterrepräsentanz und geringe Differenzierung des Einzelhandels im internationalen Vergleich. Die schnelle Entwicklung und Differenzierung des Einzelhandels gehört zu den wichtigsten Transformationsphänomenen des postsowjetischen Moskau (WENDINA 1997, Rudolph 1997). Die Differenzierung ist mit einer Belebung der alten, vorrevolutionären Geschäftsstraßen, an denen sich sehr teure und exklusive Boutiquen der Modebranchen ansiedeln, verbunden. Zunehmend treten in Moskau Formen des Einzelhandels auf, die für westeuropäische Stadtre- gionen typisch sind. Dazu gehören auch die Fachund Verbrauchermärkte in der Nähe der Fernverkehrsstraßen und Autobahnen in der Peripherie der Stadtregion. Dies ist mit einer Entwicklung neuer Standorttypen und mit einer weiteren Internationalisierung des Einzelhandels verbunden. Anzeichen hierfür sind das Auftreten großer internationaler Fachmärkte und Supermarktketten in Moskau. Die Eröffnung von Shopping-Zentren in den peripheren Stadtgebieten, in denen bisher die Kioskmärkte dominierten, war einer der wichtigsten Trends des Jahres 1999, auch wenn die Ausstattung Moskaus mit Einzelhandelsflächen außerhalb des Zentrums bisher noch weit hinter europäischen und amerikanischen Durchschnittswerten zurücksteht. 1999 betrug die Einzelhandelsfläche ca. $0,5 \mathrm{~m}^{2}$ pro Einwohner.

Die räumliche Strukturierung des Moskauer Einzelhandels unter Marktbedingungen lässt eine für marktwirtschaftliche Großstädte typische Struktur und Hierarchie von Lagequalitäten des Einzelhandels erkennen. Charakteristisch ist die unmittelbare Nachbarschaft unterschiedlicher Standortqualitäten: einerseits linienhafte Strukturen des Einzelhandels und kleinere, exklusive Bereiche mit einem hohen Anteil teurer Boutiquen in der Innenstadt, andererseits die Entwicklung des autoorientierten großflächigen Einzelhandels an der Peripherie. Der hohe Anteil Moskaus am gesamtrussischen Einzelhandelsumsatz von fast $29 \%$, die dynamische Investitionsentwicklung und die zunehmende Internationalisierung des Moskauer Einzelhandels deuten darauf hin, dass innerhalb Russlands bisher lediglich die Moskauer Region als interessanter und sicherer Markt angesehen wird. Dabei profitiert die Stadt außerdem von ihrer entwickelten Verkehrsinfrastruktur und der schnellen Erreichbarkeit im Vergleich zu anderen Regionen Russlands. Es ist wahrscheinlich, dass sich die räumlichen Strukturen des Einzelhandels in Moskau zunehmend denen west- und mitteleuropäischer Stadtregionen angleichen werden, gleichzeitig bedeutet dies jedoch eine weitere ökonomische Polarisierung Moskaus gegenüber anderen russischen Stadtregionen.

Das Stadtzentrum ist Schwerpunkt der öffentlichen und privaten Investitionstätigkeit, das den Ansprüchen der Managementfunktionen und ergänzenden Dienstleistungen sowie den Interessen der neuen «Business-Eliten» und ausländischer Geschäftsleute, d.h. den Anforderungen einer neuen tertiären Ökonomie, gerecht werden soll. Die hohe Dichte spezialisierter, wirtschaftsnaher und konsumorientierter Dienstleistungen an differenzierten Standorten bildet ein Potenzial zur Zentralisierung von Kapitalströmen in der Moskauer Innenstadt - ein Effekt der Selbstverstärkung der räumlich selektiven Konzentration des ökonomischen Wachstums. Die mit der Citybildung verbundene Standortdifferenzierung ist im Stadtzentrum am weitesten fortgeschritten. Teilweise ist dabei eine Rückkehr 


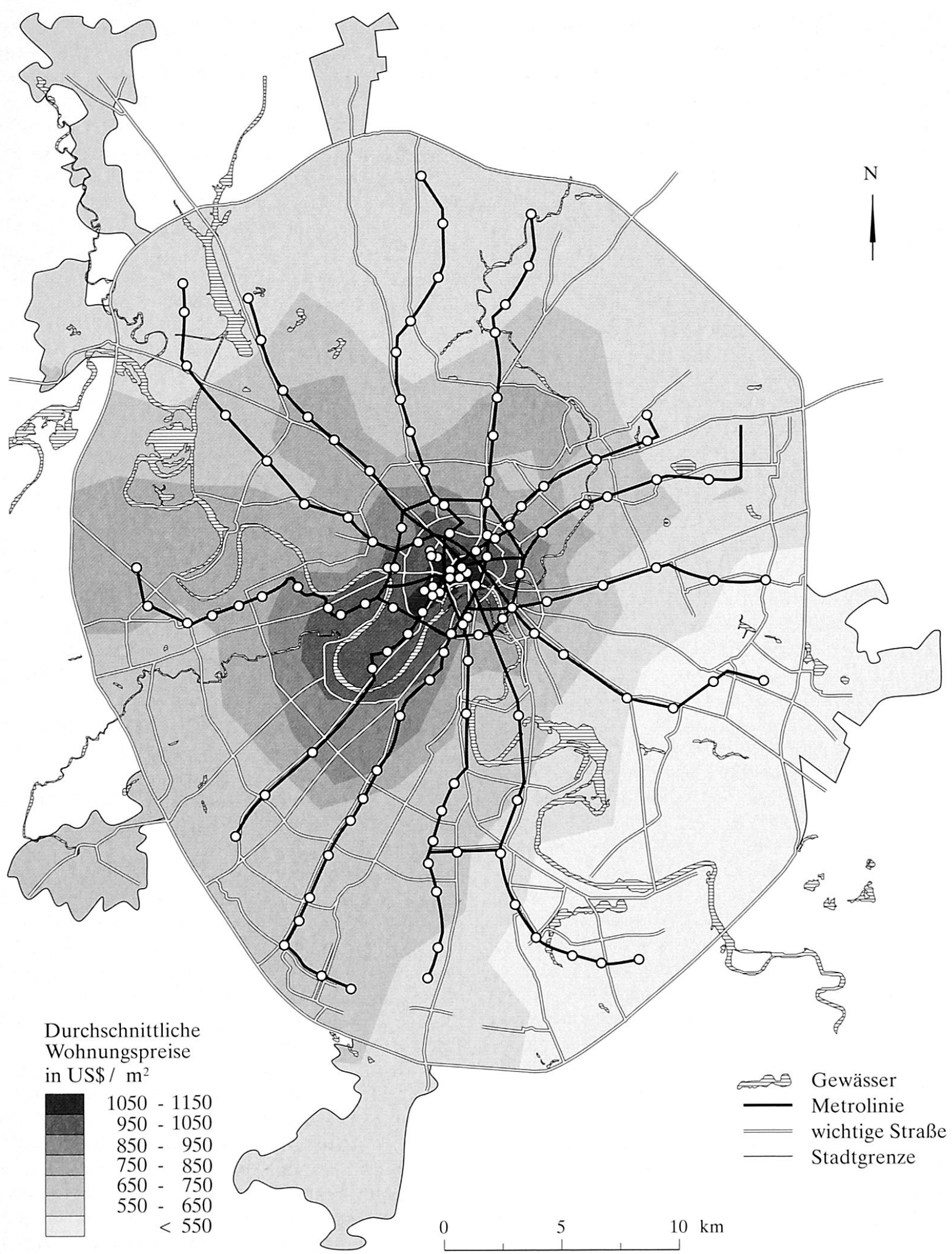

Abb. 1: Durchschnittliche Wohnungspreise in Moskau 1999 Average rents in Moscow in 1999

Prix moyens des logements à Moscou en 1999

Quelle: Monitoring rynka nedwischimosti, Nr. 1; Kartographie: R. Richter, S. Dutzmann, Institut für Länderkunde 
funktionaler Muster der vorrevolutionären Zeit zu beobachten (LENTZ 1997, RUDOLPH 1997).

Die Verlagerung des Mechanismus der räumlichen Strukturierung auf die Ebene der Kapitalverwertung widerspricht nicht dem in der zweiten Hälfte der 1990er Jahre stärker werdenden administrativen Einfluss auf die Stadtentwicklung in Moskau. Die Moskauer Administration ist der ausschließliche Grundeigentümer des Grund und Bodens, sie verfügt gleichzeitig über den weitaus größten Teil des Gebäudebestandes. Sie kontrolliert eine der größten FinanzIndustrie-Gruppen des Landes, zu der Banken, Industriebetriebe, Telekommunikations- und Medienunternehmen gehören. Die Moskauer Machtgruppen zählen zu den einflussreichsten regionalen Eliten der Russischen Föderation. Insbesondere die autoritäre Rolle des Bürgermeisters JuRi LushKow, einer herausragenden Persönlichkeit der russischen Politik mit architektonischen und städtebaulichen Ambitionen, sei hierbei hervorgehoben. Seit etwa $1996 \mathrm{nahm}$ die Kontrolle der Stadtentwicklung und der Einfluss auf das Baugeschehen bis hin zu Detailfragen der Architektur durch den Lushkow-Apparat sehr zu. Nicht allen neuen Projekten liegen rein marktwirtschaftliche Motivationen zugrunde, sie sind im Kontext der Tradition eines «ideologisierten» Städtebaus zu sehen.

\section{Budapest - Donaumetropole und Tor nach Siidosteuropa}

Mit knapp 2 Mill. Einwohnern innerhalb der Stadtgrenzen und einer Agglomerationsbevölkerung von insgesamt etwa 2,5 Mill. Einwohnern ist Budapest die unbestrittene Primate City Ungarns und die größte Stadt im östlichen Mitteleuropa. Budapest zog zwischen 1989 und 1996 über die Hälfte der ausländischen Direktinvestitionen in Ungarn an (Dingsdale 1999). Enyedi (1999) sieht die reale Chance einer Rückgewinnung der traditionellen Rolle Budapests als gateway zum südöstlichen Europa.

Die Wende zur Marktwirtschaft verlief in Ungarn effektiver und weniger abrupt als in anderen Staaten des östlichen Europa, da sich schon in der Zeit des «Gulaschkommunismus» des liberalen Parteiführers KÁDÁR während der 1970er und 80er Jahre marktwirtschaftliche Elemente einer «zweiten Wirtschaft» herausgebildet hatten. In Budapest war die ökonomische Restrukturierung der Nachwendezeit trotzdem von starken Deindustrialisierungsprozessen geprägt. Die Zahl der Arbeitsplätze in der Industrie reduzierte sich in der ersten Hälfte der 1990er Jahre um 50\% (ENYEDI 1999, KISS 1997). Der Rückgang des produzierenden Gewerbes hat jedoch nicht zu der befürchteten Massenarbeitslosigkeit geführt, da das Wachstum des tertiären Sektors die Arbeitsplatzverluste im produzierenden Bereich weitgehend ausgleichen konnte. 1999 lag die Arbeitslosigkeit in der Budapester Region bei nur 5,2\% (EuropäISCHE Kommission 2001). Budapest entwickelt sich zunehmend zu einer Dienstleistungsmetropole. Dies hatte eine wachsende Nachfrage nach modernen Büroflächen zur Folge, die zu einer starken Neubautätigkeit führte. Allein zwischen 1990 und 1993 wurden 63 neue Bürohäuser mit einer Gesamtfläche von über $300.000 \mathrm{~m}^{2}$ errichtet (LichTENBERGER 1997). Die neuen Bürogebäude konzentrieren sich im Stadtzentrum und tragen hier entscheidend zur Citybildung bei. Im zentralen Bezirk ist eine deutliche Verdichtung der existierenden Baustruktur durch Lückenbebauungen feststellbar. Bereits Mitte der 1990er Jahre waren in diesem Bereich fast alle Baulücken geschlossen (DingsDale 1999). Zahlreiche alte Industriestandorte - vor allem in Innenstadtrandlagen - wurden in Büro- oder Einzelhandelsflächen umgewandelt. Beispielhaft für diese Entwicklung steht das Einkaufszentrum Duna Plaza mit $42.000 \mathrm{~m}^{2}$ Einzelhandelsflächen.

Obwohl die städtebaulichen und sozioökonomischen Voraussetzungen für Gentrifizierungsprozesse auch in Budapest vorliegen (Kovács 1994), hat eine Aufwertung der Innenstadt durch Zuwanderung statushöherer Bevölkerungsgruppen bisher nur in sehr begrenztem Umfang stattgefunden (WIESSNER \& KovÁCS 1995). Als Hauptgrund ausbleibender Gentrifizierung führt WIESSNER (1997) an, dass bisher kein grundlegender Wertewandel zugunsten eines urbanen Lebensstils neuer Mittelschichten feststellbar sei. So werden sich die sozialräumlichen Strukturen «wohl auf längere Sicht von westlichen Mustern unterscheiden» (WIESSNER 1997).

Nach der Wende kam es zu einer umfangreichen Privatisierung des öffentlichen Wohnungsbestands durch Verkauf an die bisherigen Mieter. Resultat dieser politisch gewollten Wohneigentumsbildung ist einerseits eine soziale Stabilisierung vieler innerstädtischer Wohnquartiere durch den hohen Anteil selbstnutzender Eigentümer, andererseits jedoch eine Konzentration von sozial schwachen Bevölkerungsgruppen in den Restbeständen der öffentlich verwalteten Wohnungen und in Stadtvierteln mit weniger hohem Wohnwert. Armutsviertel bilden sich vor allem in den südöstlichen und östlichen Innenstadtrandgebieten. ENYEDI (1999) konstatiert hier bereits die Herausbildung ethnischer Ghettos der Sinti und Roma. Insgesamt hat die Privatisierung zu einer verstärkten Segregation der Bevölkerung geführt.

Die Entwicklung der Budapester Peripherie wurde in den 1960er und 1970er Jahren vor allem durch Zuwanderer geprägt, die aufgrund einer administrativen Zuzugssperre nicht in der Stadt selbst siedeln konnten und sich deshalb in den Umlandgemeinden niederließen. Suburbanisierungsprozesse «nach westlichem Muster» setzten jedoch schon in den 1980er Jahren ein, da in dieser Zeit bereits der Erwerb von privatem, neu gebautem Wohneigentum in größerem Umfang möglich war (WIESSNER 1997). Die 
Bevölkerung von Budapest verringerte sich in der Periode 1990-1996 um 130.000 Personen, davon sind etwa $40 \%$ durch Abwanderung ins Umland verursacht. Die mittelstandsorientierte Bevölkerungssuburbanisierung konzentriert sich vor allem auf die bergigen, landschaftlich attraktiveren Gebiete im nördlichen und westlichen Umland. Ein grundlegendes Merkmal der postsozialistischen Suburbanisierung in der Budapester Agglomeration ist jedoch, dass auch ältere und sozial schwächere Bevölkerungsgruppen ins Umland ziehen. Insbesondere aufgrund der steigenden Lebenshaltungskosten in der Hauptstadt wandern sie in die weniger attraktiven Gemeinden der suburbanen Zone ab. Die Einzelhandels- und Gewerbesuburbanisierung hat erst zeitlich verzögert eingesetzt und ist seit Mitte der 1990er Jahre beschleunigt (BURDACK \& HerferT 1998). Ein größerer neuer ökonomischer Wachstumsraum in der Peripherie ist im Kreuzungsbereich der Autobahnen M1/M7 (der Autobahn nach Wien und Bratislava) mit dem bestehenden Teilstück der geplanten Ringautobahn M0 bei Budaörs und Törökbalint entstanden.

\section{Jekaterinburg - Industriezentrum des Ural}

Die Stadt Jekaterinburg im östlichen Ural entwickelte sich im 19. Jh. zum Zentrum der Uralindustrie und zu einem Handels- und Finanzzentrum, dessen Einfluss weit nach Sibirien reichte. Die spezifisch städtischen Funktionen, die Geschäfts- und Handelshäuser, die Verwaltungs- und Bildungseinrichtungen konzentrierten sich auf einen relativ kleinen Teil der städtischen Fläche, während die Mehrheit der Wohngebäude aus niedriggeschossigen Holzbauten bestand. In der Sowjetunion wurde die Stadtregion zu einer der wichtigsten Industrieagglomerationen, insbesondere zu einem führenden Zentrum der Rüstungsindustrie, des Maschinenbaus und der Produktion von Ausrüstungen für den Bergbau und die Metallindustrie (RAN-UO 1993). Nach Nefjodowa \& Treiwisch (1994) gehören die Industrieregionen des Ural einem stark überindustrialisierten Typ an - mit einer einseitigen Ausrichtung auf die Grundstoff- und Schwerindustrie, geringer Branchendiversifizierung und einem hohen Anteil des militärindustriellen Komplexes. Das stark entwickelte industrielle Potenzial der Industriezentren des Ural wies keine diesem Entwicklungsstand adäquate Entwicklung der technischen und sozialen Infrastruktur auf.

Städtebaulich war die Stadt während der sowjetischen Zeit durch eine extensive Flächenentwicklung gekennzeichnet, die insbesondere auf den jenseits der inneren Industriezonen gelegenen Territorien riesige Großwohnsiedlungen entstehen ließ. Das Stadtzentrum, das heute durch das Gebiet der vorrevolutionären Stadt definiert ist, wurde städtebaulich weitgehend durch die Bebauung der 1920er bis 1950er Jahre überprägt. Nur in wenigen Bereichen des alten
Geschäftszentrums ist die vorrevolutionäre Bebauung erhalten.

Obwohl Anfang der 1990er Jahre dramatische Deindustrialisierungsprozesse einsetzten, gelang neben der enormen Ausweitung des tertiären Sektors sowohl im formellen als auch im informellen Bereich eine Konsolidierung der wirtschaftlichen Entwicklung. Der Rückgang der Industrieproduktion war im russischen Maßstab überdurchschnittlich und schwächte sich in der zweiten Hälfte der 1990er Jahre ab. Die Überindustrialisierung der Stadt mit einer Dominanz der metallverarbeitenden Industriezweige, deren Einbindung in den Rüstungssektor und der ausgeprägte Monopolisierungsgrad der Betriebe sind die hauptsächlichen Ursachen der strukturellen Probleme. Erst mit der Abwertung des Rubels infolge der Finanzkrise vom August 1998 und der daraus resultierenden Verteuerung von Importwaren setzte ein Wachstum der industriellen Produktion in Jekaterinburg ein. Einige Entwicklungen deuten auf das substanzielle Fortbestehen industrieller Kernbereiche hin. In diesem Zusammenhang kommt den in den Regionen verankerten Finanz-Industrie-Gruppen eine besondere Bedeutung zu. So gilt etwa die Privatisierung und Umstrukturierung des Jekaterinburger Unternehmens «Uralmasch", einem Hersteller von Ausrüstungen für die Rohstoffindustrie, als relativ erfolgreich. Mit dem bei St. Petersburg gelegenen Unternehmen «Ishorski sawod», das Ausrüstungen für Kraftwerke produziert, wurde 1998 ein Zusammenschluss vereinbart. Durch die enge Kooperation entstand das größte russische Unternehmen des Maschinen- und Anlagenbaus, das u.a. Kernreaktoren und Anlagen für die Erdölindustrie herstellt (GöTz 1998b). Ausländische Investitionen in den produzierenden Sektor spielten bisher eine untergeordnete Rolle. Das Gebiet Swerdlowsk, dessen Hauptstadt Jekaterinburg ist, gehört heute zu den wirtschaftlich stärksten Föderationssubjekten mit einem hohen Exportpotenzial, insbesondere aufgrund des Rohstoffreichtums (Erdöl, Buntmetalle, Halbedelsteine).

Die entstehende soziale Polarisierung führte auch in Jekaterinburg zur Entstehung eines «elitären Segments» des Wohnungsmarktes und des Einzelhandels. Die zahlungskräftige Nachfrage nach neuen Wohnungen ist wie in Moskau auf das Stadtzentrum orientiert, insgesamt ist diese jedoch zu schwach, um flächenhafte Aufwertungsprozesse in Gang setzen zu können. Neue Apartmenthäuser werden vorwiegend auf den innerstädtischen Territorien errichtet, die bisher mit traditionellen, niedriggeschossigen Holzhäusern bebaut waren. Die Jekaterinburger Innenstadt ist daher hinsichtlich ihrer Wohnfunktionen durch eine administrativ geförderte «nachträgliche Urbanisierung» des Stadtzentrums geprägt, indem die schwach bebauten Gebiete der Innenstadt nunmehr verdichtet werden. Die Strukturmuster des statushohen Wohnens 
im Gebiet der vorrevolutionären Stadt werden durch einige sowjetische Standorte ergänzt. Dies gilt, wie in Moskau, insbesondere für die in den 1930er bis 1950er Jahren angelegten Achsen mit stalinzeitlicher Bebauung.

Während sich der stark expandierende, auf breite Konsumentenschichten ausgerichtete «gewöhnliche» Einzelhandel der Kioske und Märkte auch in Jekaterinburg in erster Linie an den vorhandenen Aktionsräumen der Bevölkerung orientiert und daher die großen Magistralen und Verkehrsknotenpunkte als Standorte bevorzugt, zeigt der elitäre Einzelhandel räumliche Präferenzen in Richtung der vorrevolutionären Geschäftsstandorte im Stadtzentrum. Dieser konzentriert sich jedoch auf wenige Straßen und Passagen, die nunmehr eine Reaktivierung als gehobene Geschäftsstandorte erfahren. Mit der Aktivierung der alten Geschäftsstraßen sind Umnutzungsprozesse von Wohnungen, Investitionen in die bauliche Substanz und technische Infrastruktur verbunden, die insgesamt zu einer Aufwertung des gesamten Umfeldes dieser Standorte führen. Im Vergleich zu Moskau sind die Prozesse der Standortentwicklung jedoch bescheiden, eine Entstehung der für Moskau typischen «Zonen des Luxus» ist bisher in Jekaterinburg nicht zu beobachten.

Die weltwirtschaftliche Integration Jekaterinburgs ist bisher nur gering entwickelt und die Nachfrage nach Büroflächen von Seiten internationaler Firmen ist vergleichsweise schwach. In Jekaterinburg, wo es auch in vorrevolutionärer Zeit keine entwickelte Geschäftscity mit übernationaler Bedeutung gegeben hat und das Stadtzentrum durch sowjetische Bebauungsstrukturen stark überprägt wurde, haben die vorrevolutionären Standorte eine wesentlich geringere Bedeutung als die sowjetischen, zumal das vorrevolutionäre Geschäftsviertel räumlich relativ eng begrenzt ist. Die Territorien mit niedriggeschossiger Wohnbebauung und Brachflächen im Stadtzentrum erscheinen in der Logik der urban land rent-Modelle als untergenutzte Flächen. Gerade hier entstanden neue «Businesszentren» als relativ isolierte Standorte, die jedoch im Gegensatz zu den traditionellen Holzhäusern einer ökonomisch adäquaten Nutzung der zentralen Lage entsprechen und den hohen Potenzialwert des Standortes widerspiegeln. Die wenigen neuen Bürogebäude sind in Jekaterinburg jedoch auf eine starke Förderung durch die staatliche Administration angesichts eines ökonomisch risikoreichen Umfeldes und weniger auf internationale Nachfrageeffekte zurückzuführen.

Insgesamt sind die postsozialistischen Strukturierungsprozesse weitaus schwächer ausgeprägt als etwa in Moskau oder St. Petersburg. Die in der russischen Literatur so bezeichnete «Krise der Stadtplanung» (WLASOWA 1998) erweist sich jedoch keinesfalls als Krise der administrativen Regulation. Vielmehr haben sich die verschiedenen Privatisierungsbehörden zu den entscheidenden Akteuren der Stadtentwicklung ent- wickelt. Die Privatisierung wird jedoch maßgeblich durch regionale und lokale Cliquen kontrolliert.

\section{Łódź - das "polnische Manchester»}

Łódź entwickelte sich im 19. Jh. von einer Kleinstadt zum Zentrum der Textilindustrie im östlichen Europa. Trotz der Ansiedlung weiterer Industriebranchen wie Maschinenbau, Chemie und Nahrungsmittel in der Nachkriegszeit war Łódź auch 1989 immer noch eine «Textilstadt». Etwa die Hälfte der 171.000 Industriearbeiter waren im Textil- und Bekleidungsgewerbe beschäftigt. Nach der Wende setzte eine massive Deindustrialisierung ein und die Zahl der industriellen Arbeitsplätze sank auf 93.000 (1995). Die Arbeitslosigkeit hatte 1992 einen Höchststand erreicht. Bis 1995 reduzierte sich die Zahl der noch produzierenden Textilfabriken in der Innenstadt von 59 auf 12 (RILEY 1997b). Der weitgehende Rückzug der Industrie aus der Innenstadt stellt ein Nachholen der Entwicklung westlicher Städte im "Zeitraffer» dar. Als die planwirtschaftlich bedingten Entwicklungsbarrieren fielen, setzte sich auch in Łódź ein betrieblicher Strukturwandel durch, der zu einer Bevorzugung der produzierenden Standorte an der städtischen Peripherie führte. Der Großhandel ist der wichtigste Nachnutzer vormaliger Industrieflächen in der Innenstadt.

Die in der Nachkriegszeit entstandenen sozialistischen Industriekomplexe am Stadtrand, wie etwa ZabieniecTeofilów, bilden heute einen Industriegürtel um die Stadt. Es sind vor allem diese peripheren Industriegebiete, die als postsozialistische Produktionsstandorte bestehen blieben.

Mitte der 1990er konnten sich viele kleinere und mittlere Bekleidungs- und Textilfirmen durch Ausnutzung von Marktnischen und flexible Produktionsmethoden auf dem Markt etablieren. Es entstanden außerdem zahlreiche Firmen im Sektor der unternehmensorientierten Dienstleistungen (producer services), vor allem Marktforschung, Werbung, EDV, Rechtsberatung und Finanzdienstleistungen, auf die besonders die kleineren und mittleren Unternehmen angewiesen sind (RILEy 1998). Bedeutsam für den Aufschwung des gewerblichen Sektors ist auch die aktive Rolle regionaler Institutionen. Ein Resultat der Dezentralisierung der ehemals zentralstaatlichen Wirtschaftsförderung auf die Ebene der Wojewodschaften war die Einrichtung einer regionalen Entwicklungsgesellschaft. Die Kompetenzverlagerung auf die regionale Ebene ermöglichte hier schnellere Förderentscheidungen. Zur Stabilisierung des Arbeitsmarkts trägt jedoch auch bei, dass der Zentralstaat viele formell privatisierte Großbetriebe weiterhin kontrolliert. Mehr als die Hälfte der Industriebeschäftigten sind weiterhin in Betrieben tätig, die finanziell vom Staat gestützt werden und eher staatswirtschaftliche Strukturen als privatisierte Betriebe darstellen.

Im Stadtzentrum lässt sich eine deutliche Intensivie- 
rung ökonomischer Aktivitäten beobachten. Auffällig ist die Wiederbelebung des Einzelhandels in der traditionellen Hauptgeschäftsstraße, der Piotrkowska. Vor allem in jenen Abschnitten, in denen Maßnahmen zur Verkehrsberuhigung eingeführt wurden, konzentrieren sich Geschäfte mit gehobenem Warenangebot und elegantem Ambiente. Büronutzungen breiteten sich in zahlreichen Innenhöfen der Piotrkowska aus. Die Büros belegen in der Regel die Obergeschosse, die zuvor als Wohnraum genutzt wurden. Hier haben sich vor allem Rechtsanwälte, Versicherungsmakler und Wechselstuben niedergelassen. Die Nachfrage nach Büroflächen war jedoch vergleichsweise verhalten, neue Bürohäuser wurden nicht gebaut. Ein Indiz für den lediglich begrenzten Bedarf an zentral gelegenen Büros ist auch die Tatsache, dass sich noch zahlreiche produzierende Kleinbetriebe im Stadtzentrum befinden. Häufig belegen sie die Erdgeschosswohnungen in älteren Mietshäusern. Nahezu alle diese Betriebe gehören zum Bekleidungsgewerbe oder verwandten Branchen, wie der Lederverarbeitung (RILEY 1997a; WolANIUK 1997).

Die Entwicklung der kommunalen Selbstverwaltung hat das Engagement der Stadt zur Lösung kommunaler Probleme erheblich verstärkt. So engagiert sich die Stadt bei der Instandsetzung des jahrzehntelang vernachlässigten Altbaubestands. Gentrifizierungstendenzen durch Zuwanderung statushoher Bevölkerung sind jedoch in innerstädtischen Wohnlagen nicht festzustellen.

Der ambulante Handel «aus der Plastiktüte» - eine typische Transformationserscheinung in vielen Städten - ist inzwischen mit kleinen Märkten mit 20 bis 30 Ständen an Straßenkreuzungen, in Wohngebieten und auch in einigen größeren Märkten mit bis zu 1.000 Ständen zu finden.

Die Entwicklung der städtischen Peripherie, innerhalb und außerhalb der Stadtgrenzen, ist bisher wenig dynamisch verlaufen. Einige große Verbrauchermärkte als Filialen ausländischer Handelsketten sind in den Außenbezirken in verkehrsgünstiger Lage entstanden (RILEy, NizNiK \& Burdack 1999).

Eine Suburbanisierung der Bevölkerung «nach westlichem Muster» ist bisher noch nicht eingetreten. Łódź verzeichnete noch Mitte der 1990er Jahre einen positiven Wanderungssaldo (Województwa Łódźkiego 1996). Außerhalb der Stadtgrenze ist als einziges größeres Gewerbeprojekt in Rzgów auf einem Areal von 20 ha ein großer Textil- und Bekleidungsmarkt entstanden, der vor allem Händler aus den mittel- und osteuropäischen Ländern anzieht. Hier finden auch Produkte des lokalen Bekleidungsgewerbes Absatz.

\section{Nachholende Modernisierung oder pfadabhängige Entwicklung? - ein Resümee}

Die vergleichende Analyse der vier Untersuchungsstädte bestätigt, dass die postsozialistische Stadtent- wicklung nicht nach einheitlichem Muster verläuft, sondern erhebliche qualitative und quantitative Unterschiede aufweist. Dennoch lassen sich auch übergreifende Transformationsphänomene feststellen. Diese können mit der Wirkung politischer,ökonomischer und sozialer Prozesse, die in ihren Grundzügen im gesamten osteuropäischen, ehemals sozialistischen Raum gelten, begründet werden. Wesentlich ist in diesem Zusammenhang der tiefgreifende ökonomische Strukturwandel, der sowohl Niedergangs- als auch Wachstumsprozesse einschließt. Der strukturelle Wandel äußerte sich im Zerfall der bisherigen zentralistisch organisierten Großbetriebe und Institutionen, einer realen Deindustrialisierung und, verbunden mit einem schnellen Anwachsen kleinbetrieblicher, vielfach informeller Strukturen, einer starken Ausweitung des tertiären Sektors. Eine offensichtliche Parallele zu westlichen Stadtentwicklungen zeigt sich somit beim Prozess der Deindustrialisierung. In allen vier untersuchten Städten kam es zu einem dramatischen Rückgang der Industriebeschäftigung. Hier wurden im «Zeitraffer» Entwicklungen nachgeholt, die sich in Westeuropa über einen Zeitraum von mehreren Jahrzehnten vollzogen.

Der Grad der Integration der jeweiligen Stadt in die globalisierte Wirtschaft scheint für die Intensität der Entwicklung des tertiären Sektors, insbesondere der wirtschaftsnahen Dienstleistungen und des Büroraummarktes, ein wesentlicher Faktor zu sein. Hierbei heben sich die «Primate Cities» Moskau und Budapest deutlich ab. Die außerordentlich dynamische Entwicklung des Dienstleistungssektors äußert sich sowohl in Budapest als auch in Moskau in Citybildungsprozessen, die zu einer deutlichen Vitalisierung des Stadtzentrums führten. Die Stadtzentren erfahren hier flächenhafte Investitionsprozesse und die Formierung von Zonen des gehobenen Konsums der neuen Eliten und Mittelschichten. Citybildungsprozesse sind dagegen in Łódź und Jekaterinburg nur in sehr begrenzten Umfang zu verzeichnen. In Łódź konzentrierte sich die Aufwertung vor allem auf die historische Hauptgeschäftsstraße. Auch in Jekaterinburg orientieren sich die neuen Geschäftsfunktionen an den vorhandenen Strukturmustern der inneren Stadt, doch ist die Nachfrage nach Büroraum durch internationale Firmen vergleichsweise bescheiden.

Hinsichtlich der Wohnfunktion sind ebenfalls deutliche Entwicklungsunterschiede nachweisbar. Innerstädtische Gentrifizierungsprozesse zeigen sich vor allem in Moskau in der deutlichen Ausprägung von Gebieten «elitären Wohnens» und in begrenztem Umfang in einigen innerstädtischen Wohnlagen von Budapest.

Die deutlichste Ausdifferenzierung unterschiedlicher Entwicklungen ist jedoch in der städtische Peripherie zu beobachten. Die Budapester Peripherie wird seit den 1980er Jahren durch Suburbanisierungsprozesse nach westlichem Muster geprägt. Die Konvergenz 
des Entwicklungsweges Budapests zu den westlichen Großstadtregionen wird insbesondere am Einzelhandel deutlich: In relativ kurzer Zeit entstanden großflächige Einzelhandelsstandorte (Verbraucherund Baumärkte) an der Peripherie an den Autobahnkreuzen, die sich prinzipiell nicht von den entsprechenden, völlig internationalisierten Vorbildern westlicher Großstadtperipherien unterscheiden. In den anderen Untersuchungsstädten fehlen dagegen solche «westlichen» Suburbanisierungen. Zwar entstehen z.B. im Moskauer Umland typische Siedlungen mit den kleinen Palästen, den «Datschi» der «neuen Russen», jedoch treten diese eher als Zweitwohnsitz in Erscheinung. Von einem flächenhaft ausgeprägten, «suburbanen Wohlstandsgürtel» der Mittelschichten sind Jekaterinburg, Lódź und Moskau weit entfernt.

Insgesamt scheint somit die Ausprägung westlicher Struktur- und Entwicklungsmuster nicht nur abhängig vom «sozialistischen Erbe» der Stadt, sondern vor allem auch vom Grad der Integration der Stadt in die global economy zu sein. Budapest zeigt am deutlichsten Tendenzen eines Entwicklungsweges, der auf eine Modernisierung westlichen Typs verweist. Die sich abzeichnende gateway-Funktion für Südosteuropa und die damit verbundene Internationalisierung der Stadt stabilisiert diese Entwicklung. Dagegen weist die Entwicklung von Łódź am Ende der 1990er Jahre Ähnlichkeiten mit altindustriellen Städten des westlichen Europa auf: leerstehende und umgenutzte Fabrikgebäude, in denen sukzessiv Kleinbetriebe, der Groß- und Einzelhandel einziehen. Die weitere Entwicklung der Stadt ist jedoch eng verbunden mit der ökonomischen Stabilisierung und Konsolidierung auf nationaler Ebene. Die Voraussetzungen hierfür sind jedoch hier wesentlich günstiger (Polen ist EUBeitrittskandidat und kann daher auf entsprechende Strukturhilfen hoffen) als Jekaterinburg, wo eine Diversifizierung der Industrie in weitaus geringerem $\mathrm{Maße}$ erfolgt ist. Die geringe weltwirtschaftliche Integration der Stadt konserviert auf der einen Seite vorhandene Strukturen, auf der anderen Seite weist Jekaterinburg als Zentrum des Ural Potenziale auf, um sich stärker als gateway für die riesige und rohstoffreiche Region zwischen Europa und Asien zu entwikkeln.

Die postsozialistische Stadtentwicklung Moskaus ist durch Prozesse gekennzeichnet, die teilweise «hybride» Strukturen hervorgebracht haben. Die dynamische Investitionsentwicklung im Stadtzentrum und die Konzentration wirtschaftlicher und politischer Kontroll- und Kommandofunktionen führen zur Restrukturierung der Innenstadt und zu städtebaulichen Formen, die eine globale Orientierung erkennen lassen und «westlichen» Vorbildern folgen. Dem privilegierten Raum steht jedoch eine Peripherie gegenüber, in der bisher «improvisierte» und provisorische Strukturen dominieren. Diese Standorte reflek- tieren im Wesentlichen die Investitionsschwäche, die makroökonomische Instabilität und Unsicherheit. Es finden sich jedoch auch hier erste Anzeichen einer zunehmenden Internationalisierung. Der künftige Entwicklungsweg Moskaus wird wesentlich von der wirtschaftlichen und politischen Konsolidierung des Landes und dessen weiterer weltwirtschaftlichen Integration abhängen.

\section{Literatur}

BATER, J.H. (1980): The soviet city: ideal and reality. London: Arnold.

Burdack, J. \& G. Herfert (1998): Neue Entwicklungen an der Peripherie europäischer Großstädte. - In: Europa Regional 6/2:25-44.

DANGSChat, J. (1987): Sociospatial disparities in a «socialist» city. The case of Warsaw at the end of the 1970s'. - In: International Journal of Urban and Regional Research 11/1:37-60.

DingSDale, A. (1999): Budapest's built environment in transition. - In: GeoJournal 49/1: 63-78.

ENYEDI, G. (1994): Der Wandel postsozialistischer Städte. - In: Mitteilungen der Österreichischen Geographischen Gesellschaft 136: 53-70.

ENYEDI, G. (1999): Budapest - Gateway zum südöstlichen Europa. - In: Geographische Rundschau 51/10: 542-547.

Europäische Kommission (2001): Einheit Europas Solidarität der Völker - Vielfalt der Regionen. - Zweiter Bericht über den wirtschaftlichen und sozialen Zusammenhalt, Band 1 u. 2. - Luxemburg.

Fassmann, H. (1997): Regionale Transformationsforschung. Theoretische Begründung und empirische Beispiele. - In: MaYr, A. (Hrsg.): Regionale Transformationsprozesse in Europa. Festveranstaltung und Tagung anläßlich der 100-Jahr-Feier des Instituts für Länderkunde und Jahrestagung der Deutschen Akademie für Landeskunde. $-=$ Beiträge zur Regionalen Geographie 44: 30-45.

Fassmann, H. \& E. Lichtenberger (1995) (Hrsg.): Märkte in Bewegung - Metropolen und Regionen in Ostmitteleuropa. - Wien, Köln, Weimar: Böhlau.

FRENCH, R.A. \& F.E.I. HAMILTON (1979): The socialist city: spatial structure and urban policy. - Chichester, New York: John Wiley.

GöTz, R. (1998a): Theorien der ökonomischen Transformation. - In: Osteuropa 4/1998: 339-347.

GöTz, R. (1998b): Das «andere Rußland». Unternehmerischer Erfolg in der russischen verarbeitenden Industrie. - = Berichte des Bundesinstitutes für ostwissenschaftliche und internationale Studien 25/1998, Köln.

GRIMm, F.-D. (1994): Zentrensysteme als Träger der Raumentwicklung in Mittel- und Osteuropa. - = Beiträge zur Regionalen Geographie 37, Leipzig.

HäUSSERMANN, H. (1997): Von der sozialistischen zur 
kapitalistischen Stadt. - In: KovÁcs, Z. \& R. WIESSNER (Hrsg.): Prozesse und Perspektiven der Stadtentwicklung in Ostmitteleuropa. - = Münchner Geographische Hefte 76: 21-31.

Karger, A. \& F. Werner (1982): Die sozialistische Stadt. - In: Geographische Rundschau 11/1982: 519-528.

KISS, E. (1997): Budapest - industrieller Wandel und seine Auswirkungen auf die Stadtstruktur. - In: Europa Regional 5/4: 23-30.

KLEIN, M. (1995): Die Transformation von Ordnungen in der dualistischen Theorie und in der neuen Politischen Ökonomie. - Köln: Boterman und Boterman.

Kolossow, W.A., Wendina, O.I., Borodulina, N.A., Seredina, E.W., Fedorow, D.R. \& W.W. Klimanow (1998): Sosdanije nowoi predprinimatelskoi sredy w Moskwe: osnownyje tendenzii i protiworetschija. [Die Entstehung eines neuen unternehmerischen Milieus in Moskau: Tendenzen und Widersprüche]. - In: Iswestija Rossiskoi akademii nauk. Seria geografitscheskaja 5/1998: 95-109.

KovÁcs, Z. (1997): Transformation of the housing markets in Budapest, Prague and Warsaw. - In: Kovács, Z. \& R. Wiessner: Prozesse und Perspektiven der Stadtentwicklung in Ostmitteleuropa. - = Münchner Geographische Hefte 76: 245-256.

KovÁcs, Z. \& R. Wiessner (1999): Stadt- und Wohnungsmarktentwicklung in Budapest. Zur Entwicklung innerstädtischer Wohnquartiere im Transformationsprozeß. - = Beiträge zur Regionalen Geographie 48, Leipzig.

LENTZ, S. (1997): Cityentwicklung in Moskau - zwischen Transformation und Globalisierung. - In: Zeitschrift für Wirtschaftsgeographie 2-3/1997: 110-122.

LenTZ, S. (2000): Die Transformation des Stadtzentrums von Moskau. - In: Geographische Rundschau 7-8/2000: 11-18.

LiChTENBERGER, E. (1995): Die Entwicklung der Innenstadt von Budapest zwischen City- und Slumbildung. In: Erdkunde 49: 138-151.

Nefjodowa, T. \& A. Treiwisch (1994): Raiony Rossii i drugich ewropeiskich stran s perechodnoi ekonomikoi w natschale 90-ch. [Die Gebiete Russlands und anderer europäischer Länder mit einer Transformationsökonomie zu Beginn der 1990er Jahre]. - Moskau: Russische Akademie der Wissenschaften.

RAN-UO (Rossiskaja akademija nauk - Uralskoje otdelenije/Institut ekonomiki) (1993): Regionalny aspekt priwatisazii. [Der regionale Aspekt der Privatisierung]. - Jekaterinburg: unveröffentlichtes Manuskript, Russische Akademie der Wissenschaften, Sektion Ural, Institut für Ökonomie.

Riley, R. (1997a): Retail change in post-communist Poland with special reference to Ulica Piotrkowska, Łódź. - In: Geography 82/1:27-37.

RiLey, R. (1997b): Central area activities in a post- communist city: Łódź, Poland. - In: Urban Studies 34/3: 453-70.

RILEY, R. (1998): Manufacturing and producer services in the Łódź region of Poland. - In: European Policy and Research 5/1: 43-52.

Riley, R., NizNiK, A. \& J. BuRdack (1999): Łódź:Transformation einer altindustriellen Stadt in der postsozialistischen Periode. - In: Europa Regional 1/1999: 22-32.

Rudolph, R. (1997): Citybildung in Moskau - Prozesse der funktionalen Differenzierung. - In: Europa Regional 3/1997: 29-37.

Rudolph, R. \& S. LentZ (1999): Segregationstendenzen in russischen Großstädten: Die Entwicklung elitärer Wohnformen in St. Petersburg und Moskau. In: Europa Regional 2/1999: 27-40.

SCHLÖGEL, K. (1997): Operation Mitte - Die Wiederbelebung osteuropäischer Stadtzentren. - In: Stadtforum 24, 2/1997: 10-13.

Sмiтh, D.M. (1989): Urban inequality under Socialism. Case studies from Eastern Europe and the Soviet Union. - Cambridge: Cambridge University Press.

Stadelbauer, J. (1996): Moskau - Post-sozialistische Stadt im Transformationsprozeß. - In: Geographische Rundschau 2/1996: 113-119.

SzelenYI, I. (1996): Cities under socialism - and after. - In: Andrusz, G., Harloe, M. \& I. Szelenyi (Hrsg.): Cities after Socialism. Urban and Regional Change and Conflict in Post-Socialist Cities. - Oxford: Blackwell: $286-317$.

Wendina, O. (1997): Moskwa 90-ch: w poiskach nowoi logiki gorodskowo raswitija. [Moskau in den 90er Jahren: auf der Suche nach einer neuen Logik der städtischen Entwicklung]. - In: Geografija 39, 10/1997: 1-7.

Wiessner, R. (1997): Sozialräumliche Polarisierung der inneren Stadt in Budapest. - In: KovÁcs, Z. \& R. WIESSNER: Prozesse und Perspektiven der Stadtentwicklung in Ostmitteleuropa. - = Münchner Geographische Hefte 76: 189-201.

Wlasowa, N. Ju. (1998): Strategitscheski podchod w gorodskom planirowanii: rossiskije perspektiwy. [Strategisches Vorgehen in der Stadtplanung: Russische Perspektiven]. - In: Uralski gosudarstwenny ekonomitscheski uniwersitet (Hrsg.) (1998): Regionalnaja politika i mestnoje samouprawlenije. [Regionalpolitik und kommunale Selbstverwaltung]. - Jekaterinburg: im Selbstverlag der Universität: 13-19.

Województwa Łódźkiego (1996): Recznik Statystyczny 1996. - Łódź.

Wolaniuk, A. (1997): Spatial and functional changes in the city centre of Łódź. - In: LiszewsKi, S. \& C. Young: A Comparative Study of Łódź and Manchester. Geographies of European Cities in Transition. Łódź: Łódź University Press: 137-58. 
Zusammenfassung: Postsozialistische Stadtentwicklungen zwischen nachholender Modernisierung und eigenem Weg

Die Großstädte Ostmittel- und Osteuropas sind seit zehn Jahren von tiefgreifenden Veränderungen betroffen, die sich im Spannungsfeld von zunehmender Internationalisierung und interner Restrukturierung der Transformationsgesellschaften vollziehen. Die neue Bedeutung der Städte als Orte, die globalen Einflüssen intensiv ausgesetzt und daher von strukturellen Wandlungsprozessen in besonderem Maße betroffen sind, wird durch die schnelle ökonomische Entwicklung einiger Metropolen, durch die Deindustrialisierung der Städte, durch das schnelle Wachstum des (formellen und informellen) tertiären Sektors, durch neuartige sozialräumliche Differenzierungen und neue Entwicklungen an den städtischen Peripherien deutlich. Der Beitrag untersucht den Wandel von Städten im östlichen Europa anhand von vier Fallbeispielen (Moskau, Budapest, Jekaterinburg und Łódź). Im Zentrum der Betrachtung steht dabei die Frage nach der Erkennbarkeit unterschiedlicher Entwicklungspfade postsozialistischer Stadtentwicklung. Die vergleichende Analyse der vier Untersuchungsstädte bestätigt, dass die postsozialistische Stadtentwicklung erhebliche qualitative und quantitative Unterschiede zwischen den verschiedenen Regionen und Stadttypen aufweist. Insbesondere in den peripheren Stadträumen ist eine deutliche Ausdifferenzierung unterschiedlicher Entwicklungen zu beobachten. Dennoch lassen sich auch übergreifende Transformationsphänomene feststellen, die aufgrund der Wirkung politischer, ökonomischer und sozialer Prozesse, die in ihren Grundzügen im gesamten osteuropäischen, ehemals sozialistischen Raum gelten, begründet werden. Neben den strukturellen Ausgangsbedingungen der «sozialistischen Vergangenheit» ist jedoch der Grad der Integration der jeweiligen Stadt in die globale Wirtschaft für die Intensität der Entwicklung postsozialistischer Funktionen und stadträumlicher Strukturen ein wesentlicher Faktor.

\section{Summary: Post Socialistic Urban Development between «Catching Up» and «Own Path»}

The large urban centres of Central Eastern Europe and Eastern Europe have been affected by profound change during the last ten years, a change taking place within the difficult context of fulfilling international market requirements and the structural demands of a society undergoing political transformation. The new relevance of cities as places strongly exposed to global influences and thus particularly affected by structural transformations can be seen in the rapid economic development of some of the metropoles. The de-industrialisation of certain cities, the fast growth of both the formal and informal elements of the tertiary sector, the appearance of new socio-spatial differentiation and new development trends on the urban periphery are further signs of the new development situation many cities find themselves in. This article investigates the transformation of cities in Eastern Europe using four study cases (Moscow, Budapest, Ekaterinaburg and Lódz). The central issue is whether different paths of development may be recognised. The comparative analysis of these four cities confirms that strong differences between the regions and urban types may be observed. Particularly in peripheral urban spaces strong differences between recorded development paths were noticed. However, due to the influence of political, economic and social processes taking place in the whole of the former socialistic region of Eastern Europe common phenomena of transformation could also be detected. Apart from the structural basis of the socialistic past, it is in particular the degree to which a city has been integrated into the global economy that has influenced the intensity of development of postsocialistic functions and urban structures.

\section{Résumé: Evolutions urbaines postsocialistes entre le rattrapage de la modernité et une voie propre}

Les grandes villes d'Europe centrale de l'Est et d'Europe orientale sont concernées depuis dix ans par des transformations profondes qui se réalisent dans une ambiance de tension par suite de l'internationalisation et de la restructuration interne des sociétés postsocialistes. La nouvelle signification des villes en tant que lieux exposés de façon intense aux influences de la globalisation et, par là-même, affectés tout particulièrement par des processus de mutation structurelle, devient très apparente aux périphéries urbaines par le rapide développement économique de certaines métropoles, la désindustrialisation des villes, la montée rapide, formelle et informelle, du secteur tertiaire, de nouvelles formes de différenciation socio-spatiales et de transformation. La présente contribution analyse la mutation de villes en Europe de l'Est à partir de quatre études de cas: Moscou, Budapest, Iékaterinbourg et Łódź. La question des critères d'évaluation de l'évolution différienciée du développement urbain postsocialiste est au centre de notre interrogation. L'analyse comparée des quatre villes soumises à l'étude confirme que l'évolution urbaine postsocialiste est marquée par des différences qualitatives et quantitatives sensibles entre les divers types urbains et régions. La différenciation est notamment très nette dans les espaces urbains périphériques où nous pouvons observer des évolutions variées. Il est néanmoins possible de constater qu'il existe des phénomènes de transformation postsocialiste de portée générale fondés sur des processus d'ordre politique, économique et social suscités par les anciennes structures collectivistes et valables pour l'ensemble de l'espace postsoviétique de l'Europe de l'Est. Parallèlement aux conditions structurelles initiales inhérentes au "passé socialiste», le 
degré d'intégration de chaque ville concernée dans la globalisation économique constitue toutefois un facteur essentiel en ce qui concerne l'intensité du développement des fonctions postsocialistes et des structures spatiales urbaines.

Prof. Dr. Joachim Burdack, Dr. Robert Rudolph, Institut für Länderkunde, Universität Leipzig, Schongauerstrasse 9, D-04329 Leipzig.

e-mail:

J_Burdack@ifl-leipzig.de

R_Rudolph@ifl-leipzig.de

Manuskripteingang/received/manuscrit entré le 3.7.2001

Annahme zum Druck/accepted for publication/accepté pour l'impression: 14.12.2001 